\title{
A Novel Method for Low-constrained Iris Boundary Localization
}

\author{
Carles Fernández†, Dídac Pérez†, Carlos Segura †, Javier Hernando $\ddagger$ \\ $\dagger$ Herta Security, S.L. Barcelona, Spain \\ $\ddagger$ Universitat Politècnica de Catalunya. Barcelona, Spain \\ didac.perez@hertasecurity.com
}

\begin{abstract}
Iris recognition systems are strongly dependent on their segmentation processes, which have traditionally assumed rigid experimental constraints to achieve good performance, but now move towards less constrained environments. This work presents a novel method on iris segmentation that covers the localization of the pupillary and limbic iris boundaries. The method consists of an energy minimization procedure posed as a multilabel one-directional graph, followed by a model fitting process and the use of physiological priors. Accurate segmentations are achieved even in the presence of clutter, lenses, glasses, motion blur, and variable illumination. The contributions of this paper are a fast and reliable method for the accurate localization of the iris boundaries in low-constrained conditions, and a novel database for iris segmentation incorporating challenging iris images, which has been publicly released to the research community. The proposed method has been evaluated over three different databases, showing higher performance in comparison to traditional techniques.
\end{abstract}

\section{Introduction}

An ideal biometric system for iris recognition would be desirably non-invasive for the users, capture samples in real-time, on-the-move and at significant distance, operate in variable illumination conditions and in an entirely unsupervised manner. Nevertheless, due to the inherent difficulties associated with the capture of quality images of the iris, traditionally the environmental conditions for this process have been heavily constrained: images are usually taken under near-infrared (NIR) illumination, at close distances, strictly frontal views, and demanding user collaboration.

When dealing with real, unconstrained scenarios, the obtained iris images tend to suffer from clutter, defocus, motion blur, corneal reflections, highlights and shadows, non-frontal views, and corruptions and occlusions the iris zone, such as eyelids, eyelashes, contact lenses, or glasses. This large number of problems motivates developing so- phisticated segmentation stages that determine valid regions within the captured image, prior to the extraction of the iris pattern. Iris isolation and extraction directly influences the accuracy of the final recognition process.

Recent advances in the field have brought substantial improvements regarding NIR image acquisition, making it possible to obtain quality iris images on the move and at distances up to several meters [5]. In addition, the concern for unconstrained biometrics motivated to seek for iris recognition also in visible wavelength (VW), starting with the creation of noisy VW iris image databases such as UBIRIS 1 and 2 [8]. Although VW-based techniques exploit spectral radiance and color information in noisy environments, the quality of VW images is more degraded than that of NIR images, deriving in poorer recognition results.

Typical steps for iris segmentation include (i) a coarse localization of the eye area, usually by means of the pupil (in NIR images) or the sclera (in VW); (ii) an accurate localization of the pupillary and limbic boundaries of the iris; (iii) the localization of the upper and lower eyelid boundaries, in case of overlapping with the iris; and (iv) the inclusion of specular highlights, shadows, eyelashes and other possible artifacts within the iris area into an exclusion mask, to discard such regions from further consideration.

We focus on NIR iris recognition in low-constrained environments, covering the localization of the pupil and the two iris boundaries. Unlike traditional methods, we propose a series of techniques that do not require exhaustive search, are not based on rigid models, and do not show high sensitivity to model parameters. Instead, the method estimates boundaries by energy minimization via graph cuts, and refines the initial result by means of a fitting process and the use of physiological priors on eyelid occlusion zones.

This paper is structured as follows: Section 2 explores the existing literature on iris segmentation. Section 3 describes the proposed methodology for rough pupil detection (3.2) and localization of the pupillary (3.3) and limbic (3.4) boundaries. The experimental evaluation of these techniques is provided in Section 4, and some final conclusions are drawn in Section 5. 


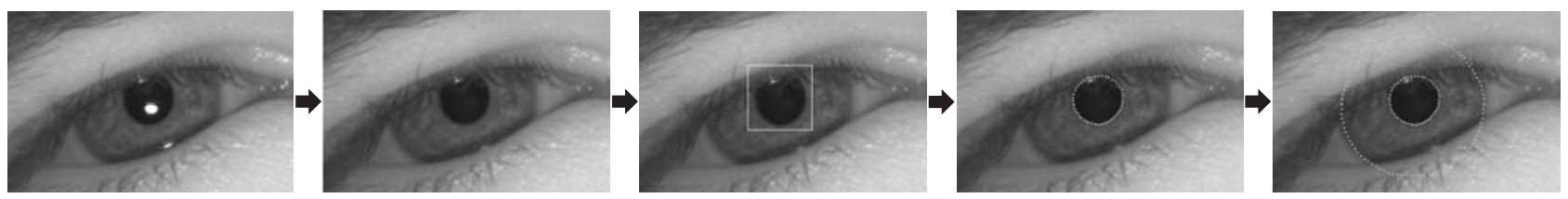

Figure 1. Pipeline of the proposed methodology. From left to right: original image, removal of specular reflection, pupil localization, pupillary boundary localization, and limbic boundary localization. Sample captures from the HID dataset.

\section{Related work}

Most of the literature on iris recognition bases its core segmentation on the techniques proposed by either Daugman [3] or Wildes [13]. Daugman's integro-differential operator estimates the localization of pupillary and limbic boundaries by detecting the most noticeable circular structures in the image. This is achieved by searching for the maximum angular integral of radial derivative over the considered domain. The path of contour integration comes defined by three parameters: the two coordinates for the circle origin within ranges $X$ and $Y$, and the radius within a range $R$, which demand the algorithm to perform an exhaustive search over the image in $X \cdot Y \cdot R$ steps.

On the other hand, the circular Hough transform is proposed by Wildes to estimate these boundaries by again detecting circular structures within the image. Typically, in order to facilitate this task, a binary edge map is first computed over the image gradient. Then, the goal is to find the triplet of two-dimensional origin coordinates and radii that best approximate such structures, which requires $N_{\text {edge }} \cdot X \cdot Y$ steps, with $N_{\text {edge }}$ the number of pixels in the edge map. Furthermore, by delimiting the range of $r$ values, the pupillary and limbic boundaries can be retrieved.

Both methods present a series of inherent drawbacks that are difficult to overcome. First of all, they require to exhaustive search over a three-dimensional parameter space, which is computationally expensive and very time consuming. In addition, these algorithms tend to fail substantially in the presence of background clutter. Finally, they both concentrate on estimating optimal parameters for hard parametric models, even though pupils and iris are sometimes far from being perfect circles or even ellipses.

Additionally, Wildes' method is based on the computation of edge maps, which is an unresolved problem of computer vision in that, so far, there is no general solution that provides satisfactory results in all cases without human intervention. Moreover, in some cases even supervised tuning fails to successfully extract the regions of interest, see Fig. 2. This is not only applicable to Wildes' method, since several recent techniques are based on the computation of edge maps. For example, [10] implements an ellipse-model fitting method, for which a large number of elliptic candidates are computed, and the best ones are selected by evalu- ating them against the result of a Canny edge detector over the original image. The incorporation of such techniques into a biometric system risks its correct behavior when moving to less-controllable scenarios.

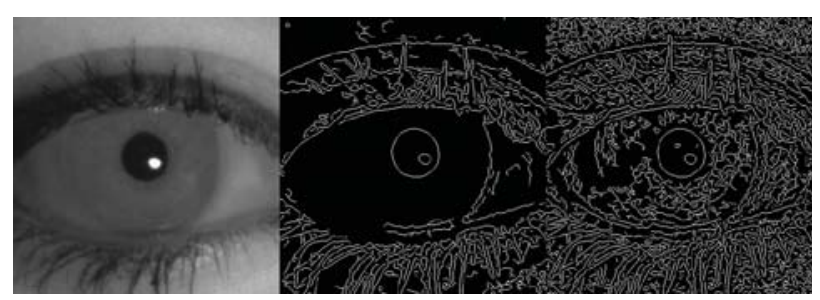

Figure 2. Sample capture from the HID dataset (left), for which Canny edge detections $(\sigma=1)$ ranging from under-segmented ( $t_{1}=0.008, t_{2}=0.02$, middle) to over-segmented results $\left(t_{1}=0.02\right.$, $t_{2}=0.05$, right) never include the complete limbic boundary.

Apart from the abovementioned methods and their derivations $[6,11]$, techniques based on elastic models and active contours have also been very actively used for iris boundary detection in the last years [12]. Such techniques present a series of limitations, such as extreme sensitivity to initialization and parameter tuning, slow convergence rates, and falling into local minima produced by noise or background clutter. Most of the currently available methods choose or combine the aforementioned strategies. For instance, [6] fuses two different algorithms, and in one of them the limbic boundaries are localized by a repeated process of Canny edge detection and Hough transform, ended with the application of an integro-differential operator.

Our solution does not rely on edge map computation, rigid-shape fitting, nor exhaustive searches over multidimensional parameter spaces, thus becoming faster and less sensitive to specific conditions.

\section{Proposed methodology}

The proposed method covers the first stages of the iris segmentation process, namely removal of specular reflections, rough localization of the pupil, and precise segmentation of the pupillary and limbic iris boundaries, see Fig. 1. 

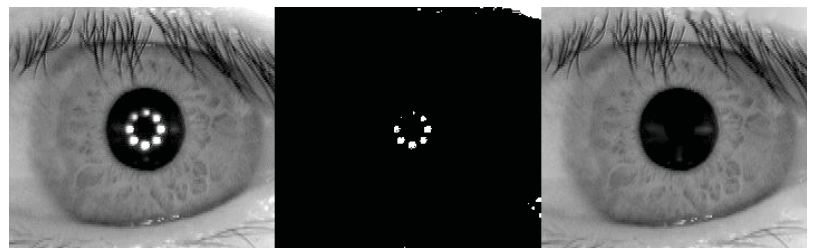

Figure 3. Removal of specularity reflections from sample S1012R03 (CASIA dataset).

\subsection{Removal of specularities}

Removing specular reflections from iris images is either tackled as a preprocessing or postprocessing step of the segmentation process. In our sequential approach it is tackled as a first step, since removing intense reflections is fundamental to localize iris boundaries, especially in the challenging cases in which the reflection invades a boundary.

Methods for reflection removal usually threshold the image to produce a mask of highlights, and then interpolate pixel intensities from neighboring regions to reconstruct masked areas. Our approach bases on the region-shrinking technique described in [9], but instead of defining a hard threshold by hand, we find it through modal analysis on the histogram of intensities. Initially, all masked pixels are listed as non-valid. Then, the intensity of each non-valid pixel having valid neighbors is linearly interpolated from them, and that pixel is removed from the list. The process repeats until the list is empty. Pixels with synthetic values will be discarded at the final recognition step.

\subsection{Coarse localization of the pupil}

The pupillary region is estimated with a morphological filter $H$, computed by removing reflections from 200 annotated images and averaging the results. The images were taken from the used databases and excluded from further tests. Candidate points are detected as proposed in [2], but again carrying out a modal analysis of the histogram to compute an adaptive threshold above which the candidates are discarded. The locations $k$ of the remaining candidates in image $I$ are scanned by the morphological filter at 10 different scales. The estimated origin and radius of the pupil are those minimizing the following test function.

$$
\underset{x, y}{\operatorname{argmin}}|I(x, y)-H(x, y)|^{2}
$$

\subsection{Pupillary boundary localization}

Having estimated the pupil origin and size, the pupillary iris boundary is precisely localized using a method based on graph cuts, and later improved using a model fitting technique, see Fig. 4. These methods are described next.

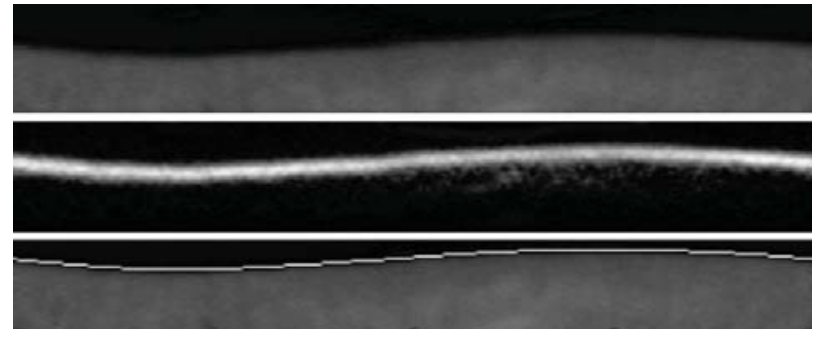

Figure 4. Top down: original polar image of the pupil region after specularity removal, enhanced vertical gradient, and estimated pupillary boundary after graph cuts and model fitting.

\subsubsection{Boundary localization via minimal cut}

As in similar early vision scenarios, the localization of iris boundaries can be posed as a multilabel energy minimization problem, and solved efficiently using graph cuts [1]. Let us consider the polar image of an eye centered at the estimated pupillary origin. We aim at finding the minimal cuts that separate the iris from the pupil and from the sclera, which would ideally correspond to the pupillary and limbic boundaries, respectively.

Let $\mathcal{S}$ be a one-dimensional ordered set of sectors defined as the regions where the target boundary shape can reside at each given angular step. On the other hand, let $\mathcal{L}$ be a set of labels that incorporate the exhaustive collection of radii in which the limbic boundary can be found. This range is statistically constrained by physiological evidence. Hence, a labeling $L$ assigns some radius label $L_{r} \in\left\{R_{\min }, \ldots, R_{\max }\right\}$ to each sector $s \in \mathcal{S}$. Then, the energy associated to a specific $L$ is:

$$
E(L)=\sum_{s \in \mathcal{S}} D_{s}\left(L_{s}\right)+\sum_{\{s, t\} \in \mathcal{N}} V_{s, t}\left(L_{s}, L_{t}\right)
$$

where $D_{s}(\cdot)$ is the data penalty function, $V_{s, t}(\cdot)$ is the interaction potential or smoothing term, and $\mathcal{N}$ is the neighborhood set of all pairs of adjacent sectors. Hence, in this approach, the horizontal coherence of the target shape is constrained by a chainwise neighborhood, whereas the vertical coherence is achieved by imposing higher compatibility to closer radius labels, and lower compatibility to distant ones.

The data penalty term has been set to the vertical gradient of the polar image, which provides information about vertical discontinuities in the intensity map. Moreover, the interaction potential, which specifies the cost associated to changing the label of a sector from radius $s$ to $t$, has been constructed by sampling a normal distribution centered at $s$ along the whole range of possible radii, see Fig. 5. Thus, the sensitivity of the whole boundary localization process is controlled by a single parameter: the standard deviation $\sigma$ of the normal distributions in the interaction potential, which 


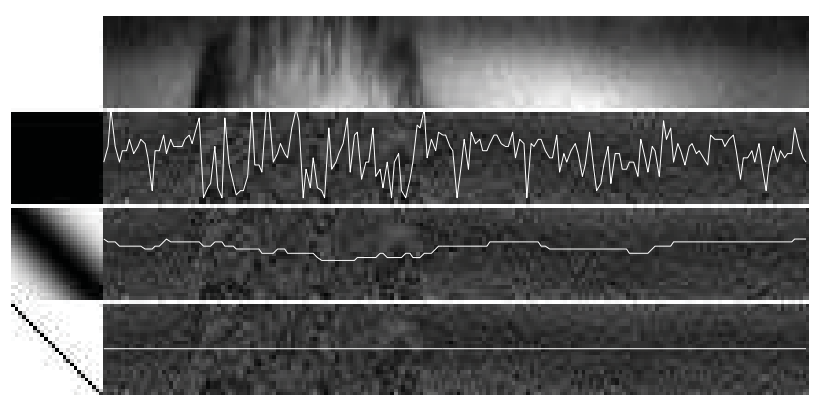

Figure 5. Top down: original polar image (HID dataset) and minimal graph cuts for $\sigma=400,8,0.4$ drawn over the computed gradient image. A visualization of the interlabel cost matrices (interaction potential) appears to the left of the gradient images.

influences the curvature of the resulting shape. The inference of the cut has average complexity of $O(|S| \log |S|)$.

\subsubsection{Model fitting}

Assuming a generic error on the estimation of the pupil center, the pupillary and limbic iris boundaries can be modeled by a generic sum of sinusoidal signals,

$$
\hat{r}(s)=\sum_{k=1}^{M} a_{k} \cos \left(\frac{2 \pi s k}{|\mathcal{S}|}+\phi_{k}\right)+b,
$$

where $s \in \mathcal{S}, M$ is the number of harmonics, and $b, a_{k}$ and $\phi_{k}$ are the search parameters to be optimized.

The optimal search parameters could be estimated by minimizing the mean squared error (MSE) between the estimated function $\hat{r}(s)$ and the set of detected boundary points $r(s)$. Nevertheless, MSE is very sensitive to outliers that are commonly caused by eyelid occlusions, and consequently, the use of MSE would require additional processes for detecting and removing outliers. In this work, we propose to use a cost function that measures the similarity between two signals while being robust to outliers. The cost function $C$ is defined as $C=-\sum_{s \in \mathcal{S}} c_{s}$, where $c_{s}=e^{-d_{s} / \lambda}$ and $d_{s}=\|r(s)-\hat{r}(s)\|$ is the Euclidean distance between the detected and estimated radius. $c_{s}=1$ when the distance is 0 , and $c_{s}$ decays rapidly to 0 when the distance increases. The decay rate is controlled by $\lambda$.

The parameters of function $\hat{r}(s)$ are optimized using Nelder-Mead (Simplex) [7], a direct search method that attempts to minimize nonlinear functions of $n$ real variables using only function values, without requiring explicit nor implicit derivative information. In the case of an ndimensional space, the simplex is characterized by the $n+1$ vectors as its vertices. At each search iteration, the function value of a new point near or within the current simplex is evaluated and compared with those of the vertices. One vertex is then replaced by the new point if the energy decreases, and this is repeated until the diameter of the sim-

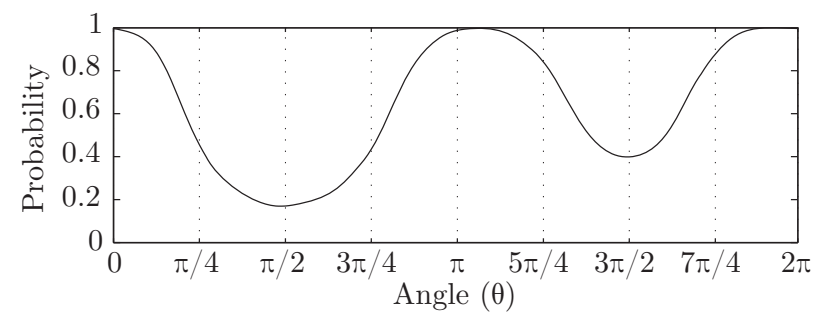

Figure 6. Probability of not observing eyelid overlapping at angle $\theta$. The upper eyelid corresponds to $\pi / 2$, the lower one to $3 \pi / 2$.

plex is lower than a given tolerance. The method does not guarantee global convergence, but is especially suitable for systems requiring temporal and computational efficiency, as opposed to any variation of the gradient descent search.

\subsection{Limbic boundary localization}

In NIR iris images, limbic boundaries are typically more challenging than pupillary ones, given the potential occlusions by the eyelids. However, this process can be eased by physiological evidence; as described in [4], the radius of the pupil varies within a range of $10-80 \%$ of the diameter of the iris. In addition, whereas it is common for the upper eyelid to notably overlap the iris region, the lower eyelid is usually less invading and presents minor to no overlapping.

\subsubsection{Eyelid occlusion priors}

In order to give more importance to those regions showing clear limbic boundaries, a probability function $\operatorname{pr}(s)$ is estimated from over 1000 eyelid annotations from different databases. This function, shown in Fig. 6, gives us information about the probability of not observing eyelid overlap at angle $s$, thus leaving out of the analysis those angular regions that are statistically prone to occlusions and artifacts.

\subsubsection{Region bounding and contour fitting}

A region of interest is placed at $[r, r+\Delta r]$ by maximizing Eq. (4) over the vertical gradient of polar image $I$.

$$
\underset{r}{\operatorname{argmax}}\left|\int_{r}^{r+\Delta r} p r(s) \cdot \frac{\partial}{\partial r} I(s, r) d r\right|
$$

Contour fitting is carried out over this region by the described graph cuts technique. Before applying model fitting to this result, the local variance of each $r(s)$ is computed on a neighborhood $L$ by $\varepsilon(s)=\sum_{k=s-L}^{s+L}|r(k)-r(s)|^{2}$.

The result of this method additionally gives information about the continuity of the signal, approaching the limbic boundary and the bad measurements, such as eyelids, eyelashes and other corrupting elements. Thus, $\varepsilon(s)$ is normalized in the range $\{0, \ldots, 1\}$ and combined with the $\operatorname{pr}(s)$ to 
Table 1. Used databases, number of annotations, and image sizes.

\begin{tabular}{ccc}
\hline Database & Number of annotations & Dimensions (px) \\
\hline MMU1 $^{1}$ & 450 & $320 \times 240$ \\
IREX $^{2}$ & 178 & $640 \times 480$ \\
HID $^{3}$ & 400 & $480 \times 480$ \\
\hline
\end{tabular}

${ }^{1} \mathrm{http}: / /$ pesona.mmu.edu.my/ ccteo/

2 NIST IREX III validation package from http://www.nist.gov/itl/ $\mathrm{iad} / \mathrm{ig} /$ irexiii.cfm, which is a subset of the ND-IRIS-0405 dataset.

${ }^{3} \mathrm{http}: / /$ research.hertasecurity.com/datasets/HID

give a global probability of a successful boundary segmentation. The output is iteratively used to improve the cost function $C$ on Eq. (5), which finally results in accurate localization of the contour points of the iris without taking into account the eyelid boundaries.

$$
C=-\sum_{s \in \mathcal{S}} c_{s} \cdot \operatorname{pr}(s)[1-\bar{\varepsilon}(s)]
$$

\section{Experimental validation}

In order to conduct experimental validation of the presented segmentation algorithm, a new database identified as HID (Herta Iris Database) was created, consisting of iris images captured under a series of unconstrained environments. These include different illumination conditions, variable distance ranges, presence of glasses and lenses, motion blur, and unfocused images, among others.

In addition, due to the lack of annotated iris segmentation resources, we manually annotated two publicly available databases, obtaining a final dataset of more than 1000 annotated irides. The HID iris database and the whole collection of annotations is available to the research community. Table 1 describes the annotated databases.

Pupil localization was evaluated in terms of distance to the annotated center. The average distances and deviations in pixels were $4.9 \pm 10.2$ for HID, $3.3 \pm 2.0$ for MMU1, and $3.5 \pm 4.8$ for IREX. The localization of pupillary and limbic boundaries was evaluated in terms of accuracy of the binary segmentation, i.e. the coincidence of pixels between the predicted binary mask for the iris region and the annotated one. Two metrics were considered: overall accuracy $(O A)$ and segmentation accuracy $(S A)$, defined by

$$
O A=\frac{T P+T N}{T P+F P+T N+F N}, \quad S A=\frac{T P}{T P+F P+F N},
$$

being $T P, T N, F P, F N$ true positives, true negatives, false positives and false negatives, respectively. $O A$ is typically overfavored, given the high values of $T N$ in an image. This happens especially for multiclass environments, but is also noticeable in the binary case. For this reason, $S A$ is increasingly been used to evaluate segmentations.

We compared our method to the two most widely used algorithms for boundary localization, [3] and [13]. We used the best publicly available implementations we found and optimized them for these datasets. Given that these techniques require exhaustive search, which involves a high computational cost, we first ran our pupil localization method and reduce their search area to a region comprising the iris. Four algorithmic variations were tested to assert the contribution of our side proposals: the core minimal cut process $(\mathrm{MC})$, the cut followed by the Simplex model fitting $(\mathrm{MC}+\mathrm{S})$, the cut with statistical eyelid priors $(\mathrm{MC}+\mathrm{P})$, and the full solution $(\mathrm{MC}+\mathrm{S}+\mathrm{P})$. All experiments were conducted on a $2.7 \mathrm{GHz}$ dual-core machine with 4 GB RAM.

Results in Table 2 show that the core graph cuts algorithm outperforms traditional techniques for boundary localization, both in terms of accuracy and computational performance. The full proposal further increases the accuracy, in exchange of lowering the efficiency to levels comparable to those of traditional techniques. The method does not depend on rigid models, is not very sensitive to the parameters, and does not search exhaustively over multidimensional spaces. Instead, a non-optimal but accurate solution is provided first, and this result is further optimized by an efficient model fitting method and the use of prior physiological data, which have been proven effective to ignore artifacts towards more precise localization.

\section{Conclusions and Future Work}

Recent advances on iris biometrics bring new challenges regarding unconstrained conditions and computational efficiency. We presented a novel iris boundary localization method to address both challenges, which is based on energy minimization by graph cuts, and empowered by pupil localization by sliding average pattern, model fitting by direct search, and defocus of irrelevant regions. These ideas do not suffer from the most important limitations of traditional methods: rigidity of the models, extreme parameter sensitivity, and exhaustive search. The solution can cope with iris images corrupted by the typical artifacts of lowconstrained environments, while obtaining accurate results in a faster and more reliable way than traditional techniques. Moreover, we have compiled and publicly released our own dataset of low-constrained NIR iris images.

The current algorithm handles slight rotations, but the shifting of priors for large rotations must be addressed in the future. Moreover, localizing iris boundaries is not the only problem of iris image segmentation. Further work includes upper and lower eyelid localization, eyelash segmentation, and identification of noisy regions.

\section{Acknowledgments}

This work has been partially funded by the Spanish project SARAI (TEC2010-21040-C02-01). We thank David Oro and Javier R. Saeta for their helpful discussions. 


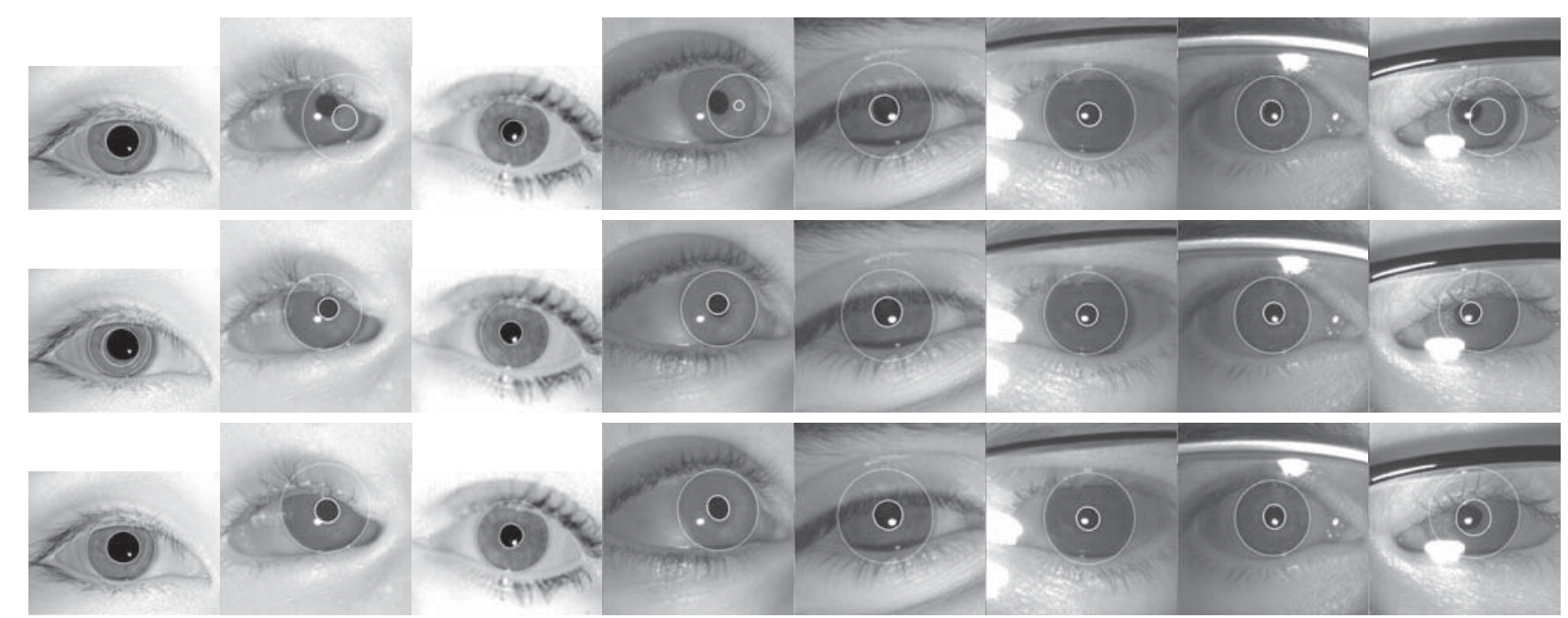

Figure 7. Examples of failure, imprecision, and success using [3] (top), [13] (middle), and our proposed method (bottom). Sample images from the used databases include presence of lenses and glasses, half-closed or non-frontal eyes, and strong highlights, among others.

Table 2. Comparative results of traditional localization methods to our core proposal based on minimal cuts $(\mathrm{MC})$ and its step variations via Simplex model fitting $(\mathrm{S})$ and eyelid priors $(\mathrm{P})$.

\begin{tabular}{|c|c|c|c|c|c|c|c|}
\hline & & [3] & [13] & MC & $\mathrm{MC}+\mathrm{S}$ & $\mathrm{MC}+\mathrm{P}$ & $\mathrm{MC}+\mathrm{S}+\mathrm{P}$ \\
\hline \multirow{3}{*}{ MMU1 } & OA & $93.4 \%$ & $91.9 \%$ & $97.5 \%$ & $97.6 \%$ & $97.6 \%$ & $97.6 \%$ \\
\hline & $\mathbf{S A}$ & $53.0 \%$ & $34.8 \%$ & $83.3 \%$ & $84.1 \%$ & $84.0 \%$ & $88.5 \%$ \\
\hline & Time & $449 \mathrm{~ms}$ & $196 \mathrm{~ms}$ & $362 \mathrm{~ms}$ & $558 \mathrm{~ms}$ & $379 \mathrm{~ms}$ & $557 \mathrm{~ms}$ \\
\hline \multirow{3}{*}{ IREX } & OA & $95.5 \%$ & $96.0 \%$ & $96.2 \%$ & $96.2 \%$ & $96.2 \%$ & $96.3 \%$ \\
\hline & $\mathbf{S A}$ & $77.9 \%$ & $72.8 \%$ & $79.5 \%$ & $79.7 \%$ & $79.5 \%$ & $80.8 \%$ \\
\hline & Time & $1044 \mathrm{~ms}$ & $816 \mathrm{~ms}$ & $471 \mathrm{~ms}$ & $667 \mathrm{~ms}$ & $471 \mathrm{~ms}$ & $669 \mathrm{~ms}$ \\
\hline \multirow{3}{*}{ HID } & OA & $95.0 \%$ & $96.1 \%$ & $97.7 \%$ & $98.4 \%$ & $94.1 \%$ & $98.7 \%$ \\
\hline & $\mathbf{S A}$ & $76.6 \%$ & $78.3 \%$ & $87.2 \%$ & $90.9 \%$ & $88.3 \%$ & $92.3 \%$ \\
\hline & Time & $1132 \mathrm{~ms}$ & $500 \mathrm{~ms}$ & $397 \mathrm{~ms}$ & $602 \mathrm{~ms}$ & $385 \mathrm{~ms}$ & $630 \mathrm{~ms}$ \\
\hline
\end{tabular}

\section{References}

[1] Y. Boykov and V. Kolmogorov. An experimental comparison of min-cut/max-flow algorithms for energy minimization in vision. IEEE TPAMI, 26(9):1124-1137, 2004.

[2] T. A. Camus and R. Wildes. Reliable and fast eye finding in close-up images. ICPR, 1:389-394, 2002.

[3] J. Daugman. High confidence visual recognition of persons by a test of statistical independence. IEEE TPAMI, 15(11):1148-1161, 1993.

[4] J. Daugman. How iris recognition works. IEEE TCSVT, 14(1):21-30, 2004.

[5] C. Fancourt, L. Bogoni, K. Hanna, Y. Guo, R. Wildes, N. Takahashi, and U. Jain. Iris recognition at a distance. In Audio- And Video-Based Biometric Person Authentication, volume 3546, pages 187-200. Springer LNCS, 2005.

[6] P. Li, X. Liu, L. Xiao, and Q. Song. Robust and accurate iris segmentation in very noisy iris images. IMAVIS, 28(2):246253,2010 .
[7] J. A. Nelder and R. Mead. Iris detection using intensity and edge information. Computer Journal, 7:308-313, 1965.

[8] H. Proença and L. Alexandre. Ubiris: A noisy iris image database. ICIAP, pages 970-977, 2005.

[9] S. Pundlik, D. Woodard, and S. Birchfield. Non-ideal iris segmentation using graph cuts. In CVPR Workshops. IEEE, 2008.

[10] W. Ryan, D. Woodard, A. Duchowski, and S. Birchfield. Adapting starburst for elliptical iris segmentation. In 2nd Int. Conf. on Biometrics. IEEE, 2008.

[11] T. Tan, Z. He, and Z. Sun. Efficient and robust segmentation of noisy iris images for non-cooperative iris recognition. IMAVIS, 28(2):223-230, 2010.

[12] M. Vatsa, R. Singh, and A. Noore. Improving iris recognition performance using segmentation, quality enhancement, match score fusion, and indexing. IEEE TSMC-B, 38(4):1021-1035, 2008.

[13] R. Wildes. Iris recognition: an emerging biometric technology. Proceedings of the IEEE, 85(9), 1997. 